\title{
Sorafenib for the treatment of unresectable hepatocellular carcinoma
}

Junji Furuse

Department of Internal Medicine, Medical Oncology, School of Medicine, Kyorin University
Correspondence: Junji Furuse

Department of Internal Medicine, Medical Oncology, School of Medicine, Kyorin University 6-20-2, Shinkawa, Mitaka-shi,

Tokyo, I8I-86II, Japan

$\mathrm{Tel}+8 \mathrm{I} 4224755 \mathrm{II}$

Fax +8I 42244 I858

Email jfuruse@ks.kyorin-u.ac.jp
Abstract: Raf kinases and vascular endothelial growth factor receptor (VEGFR) and platelet-derived growth factor receptor (PDGFR) tyrosine kinases are potential molecular targets for obtaining both anti-tumor cell progression and anti-angiogenesis effects in cancers, including hepatocellular carcinoma (HCC). Sorafenib is an oral multi-kinase inhibitor that mainly targets Raf kinases and receptor tyrosine kinases associated with angiogenesis (VEGFR-2/-3, PDGFR- $\beta$ ). A global randomized controlled trial (RCT) of sorafenib versus placebo conducted in patients with advanced HCC demonstrated the beneficial effects of the drug on the time-to-progression and overall survival. Furthermore, a RCT with a similar design to that of the global trial conducted in the Asia-Pacific region also demonstrated the efficacy of the drug. The most common treatment-related adverse events of sorafenib were found to be diarrhea, fatigue, and skin toxicity, namely, hand-foot syndromes and rash. Based on the results of the RCTs, sorafenib has been established as a standard agent for systemic chemotherapy in HCC patients with metastatic disease or transcatheter arterial chemoembolization (TACE)-refractory disease who are not suitable candidates for local treatments. The efficacy and safety of sorafenib in patients with moderate liver dysfunction have not been confirmed to date and more data are needed. Development of new therapeutic methods is needed for the treatment of advanced HCC in the future; clinical trials of sorafenib-based combination therapy, second-line therapy after sorafenib failure, and adjuvant therapy after local treatments are warranted in HCC patients.

Keywords: hepatocellular carcinoma, sorafenib, raf kinase, vascular endothelial growth factor receptor, platelet-derived growth factor receptor

\section{Introduction}

Recently, some growth factors and various signal transduction pathways have been identified, and various molecular-targeted agents have being tried for the treatment of various tumors, including hepatocellular carcinoma (HCC). These signal transduction pathways control the processes of cell proliferation and survival, and also specialized functions such as angiogenesis. Dysregulated signaling pathways contribute to malignant transformation in human cells. Sorafenib is a small-molecule multi-kinase inhibitor that inhibits kinases such as Raf kinase, vascular endothelial growth factor receptor (VEGFR), and platelet-derived growth factor receptor (PDGFR)- $\beta$ tyrosine kinases (Wilhelm et al 2004). It inhibits tumor-cell proliferation and angiogenesis and increases the rate of apoptosis in a wide range of tumor models (Gollob et al 2006; Liu et al 2006; Wilhelm et al 2006; Chang et al 2007).

HCC is the fifth most common malignancy worldwide, with approximately 500,000 new cases diagnosed each year (Parkin et al 2005). Most patients with HCC have chronic liver disease, especially liver cirrhosis, which is mainly the result of hepatitis virus infection. However, there are clearly defined differences in the etiology of HCC among different geographic regions. Hepatitis B virus (HBV) infection is very common in East and South-East Asia and Africa; more than $80 \%$ HCC patients in these countries have HBV infection (McGlynn et al 2001). On the other hand, hepatitis C virus (HCV) 
is more common in Japan, and HCV antibody is observed in $72 \%$ of HCC patients in Japan (Ikai et al 2005). The incidence of HCV infection is also increasing in the US and Europe, as is the incidence of HCC (El-Serag and Mason 1999). Various treatment modalities such as surgery, ablation, transcatheter arterial chemoembolization (TACE), and liver transplantation, are available as local therapeutic approaches for HCC. Despite the efficacy of local therapies, patients frequently develop recurrence or disease progression after these treatments. These patients are then offered systemic treatment including chemotherapy. However, although response rates of $10 \%$ to $20 \%$ have been reported, the survival benefit of chemotherapy has never been confirmed, either for singleagent or combination chemotherapy, in these patients.

A phase III, double-blind, placebo-controlled trial of sorafenib showed survival benefit in patients with advanced HCC in 2007 (Llovet et al 2008). As a result, sorafenib has been established as a standard agent for systemic chemotherapy in patients of HCC. In this paper, the mechanism of action of sorafenib and clinical trials of sorafenib for the treatment of HCC are reviewed, and future directions for the use of this drug are discussed.

\section{Mechanism of action of sorafenib}

Many of the processes involved in tumor progression, such as cell proliferation, invasion, metastasis and inhibition of apoptosis, are mediated by signaling pathways triggered by activated receptor tyrosine kinases. Ras functions downstream of several receptor tyrosine kinases, and activation of Ras signaling pathways is an important mechanism underlying the development of human cancer. Ras regulates several pathways that synergistically induce cellular transformation, including the Raf/mitogen-activated protein kinase (MAPK) kinase (MEK)/extracellular signal-regulated kinase (ERK) cascade (Hackel et al 1999; Woodburn 1999; Wilhelm et al 2004). The Raf kinases are serine/threonine protein kinases that function in this pathway as downstream effector molecules of Ras (El-Serag and Mason 1999; Wilhelm et al 2004; Llovet et al 2008). The Raf kinase family is composed of three members, namely, A-Raf, B-Raf and Raf-1. Mutations of B-Raf have been identified in various human tumors, such as malignant melanomas, colorectal cancer and thyroid papillary cancers (Davies et al 2002; Kimura et al 2003). Previous studies have reported observing Raf-1 hyperactivation in 6 of $11(55 \%)$ renal cell carcinoma (RCC) tumors and all of $30 \mathrm{HCC}$ tumors (Oka et al 1995; Hwang et al 2004). Solid tumors aberrantly secrete many different growth factors and cytokines, including VEGF and PDGF- $\beta$. The binding of these growth factors to receptors on the surface of endothelial cells activates the Raf/MEK/ERK pathways, which results in the induction of angiogenesis and lymphangiogenesis (Hood et al 2002). Thus, the Ras/Raf signaling pathway is an important mediator of tumor cell proliferation and angiogenesis in various solid tumors including HCC. Raf kinases and the VEGFR and PDGFR tyrosine kinases are potential molecular treatment targets for obtaining both antitumor cell progression and antiangiogenesis effects.

In order to discover novel therapies targeting the Ras/Raf/ MEK/ERK pathway, screening for Raf-1 kinase inhibitory activity was initiated in 1995 by Bayer and Onyx (Figure 1) (Wilhelm et al 2006). The first compound generated, 3-thienyl urea, demonstrated Raf1 $\mathrm{IC}_{50}$ (half maximal inhibitory concentration) of $17 \mu \mathrm{mol}$, and finally sorafenib achieved $\mathrm{IC}_{50}$ of $6 \mathrm{nmol}$ (Table 1) (Wilhelm et al 2004; Wilhelm et al 2006). The chemical name of sorafenib is $N$-(3-trifluoromethyl4-chlorophenyl)- $N$ '-(4-(2-methylcarbamoyl pyridin-4-yl) oxyphenyl) urea, and the structural formula is shown in Figure 1. Sorafenib was also shown to potently inhibit the wild-type-Raf, and oncogenic b-raf V600E serine/threonine kinase, pro-angiogenic receptor tyrosine kinases, c-kit, and Flt-3 in vitro (Table 1) (Wilhelm et al 2006).

The anti-tumor efficacy and mechanism of action of sorafenib was investigated in vitro on $\mathrm{PLC} / \mathrm{PRF} / 5$ and HepG2 $\mathrm{HCC}$ cells and in vivo on PLC/PRF/5 human tumor xenografts in severe combined immunodeficient mice (Liu et al 2006). Sorafenib inhibited the phosphorylation of MEK and ERK and down-regulated cyclin D1 levels in these two cell lines. Furthermore, sorafenib reduced the phosphorylation level of eIF4E and down-regulated the anti-apoptotic protein Mcl-1 in a MEK/ERK-independent manner. Sorafenib demonstrated dose-dependent tumor growth inhibition of implanted PLC/ PRF/5 tumor xenografts (Liu et al 2006). The in vivo antitumor activity of sorafenib correlated with the inhibition of MAPK signaling, which is indicative of Raf kinase inhibition, and inhibition of tumor microvessel area as measured by the reduction in CD34 staining (Liu et al 2006). These experiments showed that the anti-tumor activity of sorafenib was attributed to inhibition of tumor angiogenesis of VEGFR and PDGFR and direct effects on tumor cell proliferation/survival of Raf kinase signaling-dependent and signaling-independent mechanisms (Figure 2).

\section{Systemic chemotherapy for hepatocellular carcinoma}

Systemic chemotherapy is applied for patients with advanced $\mathrm{HCC}$ who are not suitable candidates for local treatments, 


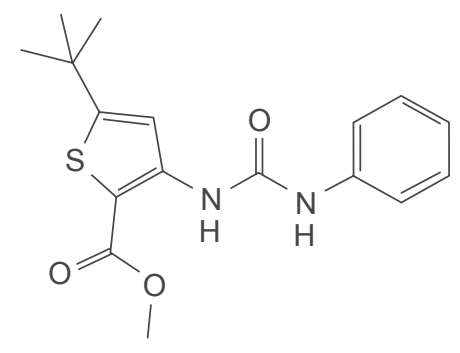

1 Raf1 $\mathrm{IC}_{50}=17 \mu \mathrm{M}$

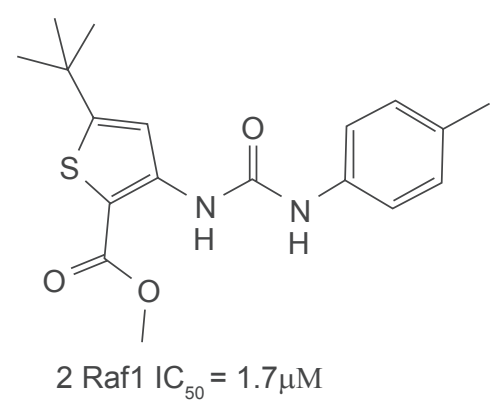<smiles>CC(C)(C)c1cc(NC(=O)Nc2ccc(Oc3ccccc3)cc2)no1</smiles>

3 Raf1 $\mathrm{IC}_{50}=1.1 \mu \mathrm{M}$

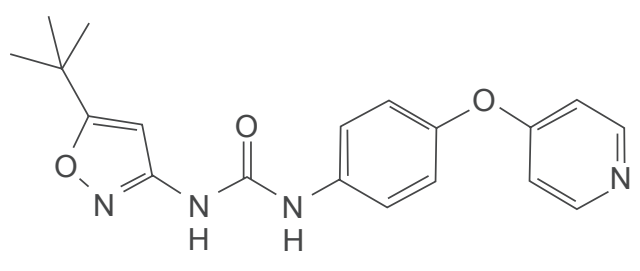

4 Raf1 $\mathrm{IC}_{50}=230 \mu \mathrm{M}$

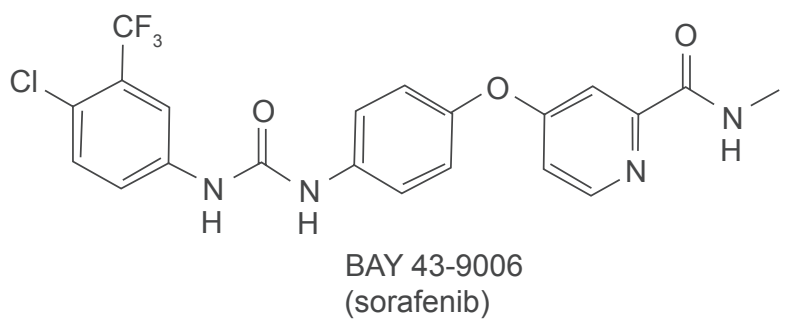

Figure I Chemical structural formulas of compounds inhibiting Rafl and sorafenib. Adapted by permission from Macmillan Publishers Ltd: Nat Rev Drug Discov, 5:835-44. @ 2006.

eg, those with distant metastasis and/or macrovascular invasion (Bruix and Sherman 2005). Refractoriness to TACE is also considered as an indication for systemic chemotherapy. Various studies on chemotherapy for HCC have indicated that anthracycline anti-tumor antibiotic agents, such as doxorubicin and mitoxantrone, constitute the basis for chemotherapy in cases of HCC (Lai et al 1988; Colleoni et al 1992). Furthermore, cisplatin and/or fluorouracil are the agents most commonly used in combination chemotherapy for HCC (Leung et al 1999; Boucher et al 2002; Patt et al 2003; Ikeda et al 2005). The response rates to combination chemotherapies, including fluorouracil/mitoxantrone/cisplatin (FMP), epirubicin/cisplatin/fluorouracil (ECF) and cisplatin/interferon $\alpha$-2b/doxorubicin/fluorouracil (PIAF), have been reported to range from $14 \%$ to $26 \%$, with the median overall survival (OS) ranging from 8.9 to 11.6 months. Doxorubicin has been considered as a referential arm in randomized clinical trials for HCC based on the results of a comparative trial between doxorubicin and supportive treatment (Lai et al 1988). Despite phase III trials indicating better responses to combination chemotherapy than to doxorubicin monotherapy, no standard chemotherapy has yet been established that can clearly prolong the survival in advanced HCC patients; eg, a recent phase III trial of doxorubicin versus PIAF failed to show any survival benefit of either drug (response rate: $10.5 \%$ for doxorubicin and 20.9\% for PIAF, $\mathrm{p}=0.058$; median OS: 6.8 months for doxorubicin and 8.7 months for PIAF, $\mathrm{p}=0.83$ ) (Yeo et al 2005). Thus, no standard regimen has been identified by large prospective clinical trials conducted until 2007, which can clearly prolong the survival in patients with advanced HCC.

\section{Rationale for use and clinical trials of sorafenib for hepatocellular carcinoma}

Hepatocarcinogenesis is a multi-step process, slowly unfolding on a background of chronic liver disease, most 
Table I In vitro inhibitory profile of sorafenib. Adapted by permission from Macmillan Publishers Ltd: Nat Rev Drug Discov, 5:835-44. (C) 2006

\begin{tabular}{ll}
\hline Kinase target & IC $_{50}$ value (nmol/L) \\
\hline Raf-I & 6 \\
BRAF wild-type & 22 \\
Oncogenic b-rafV600E & 38 \\
VEGFR-I & 26 \\
VEGFR-2 & 90 \\
Murine VEGR-3 & 20 \\
Murine PDGFR & 57 \\
FIt-3 & 33 \\
P38 & 38 \\
c-Kit & 68 \\
FGFR-I & 580 \\
ERK-I, MEK-I, EGFR, HER-2/neu, & $>10,000$ \\
IGFR-I, c-met, PKA, PKB, CDKI/ & \\
cyclin B, pim-I, PKC $\alpha$, PKC $\gamma$ & \\
\hline
\end{tabular}

often chronic hepatitis and cirrhosis. Recent insights into the biology of HCC suggest that certain signaling pathways such as the PI3K/Akt/mTOR, Ras/Raf/MEK/ERK and Wnt/ $\beta$-catenin pathways mediated by EGFR and angiogenic signaling pathways mediated by VEGFR and PDGFR play important roles in the development of HCC by promoting cell growth and survival (Avila et al 2006). Some studies have demonstrated the important roles played by MAPK/ ERK activation in the multi-step carcinogenetic process involved in human HCC, especially in the progression of HCC (Schmidt et al 1997; Ito et al 1998). Overexpression of phosphorylated MEK1/2 and MAPK in HCC cell lines was found to enhance tumor growth and survival by preventing apoptosis (Huynh et al 2003). HCC is highly related to HBV and $\mathrm{HCV}$ infection, and a relationship between expression of Raf and HCV/HBV infection has been demonstrated. Giambartolomei et al (2001) reported that the ability of HCV core proteins to directly activate the MAP kinase cascade, especially Raf-1, and to prolong its activity in response to mitogenic stimuli may contribute to the neoplastic transformation of HCV infected liver cells. Chen and Siddiqui (2007) reported that $\mathrm{HBV} X$ protein $(\mathrm{HBx})$ plays a crucial role in the viral life cycle and contributes to the onset of HCC. HBx caused mitochondrial translocation of Raf-1 kinase either alone or in the context of whole-viral-genome transfections. $\mathrm{HCC}$ is generally hypervascular and contains rich tumor vascularity, and VEGF, which is related to angiogenesis, is one of the important factors involved in the angiogenesis in HCC (Miura et al 1997; Yamaguchi et al 1998). Moreover, VEGF promotes HCC development and metastasis, and the serum VEGF level has been reported as a significant independent prognostic factor in patients with $\mathrm{HCC}$ (Chao et al 2003; Poon et al 2004). Thus, the Raf/MEK/ERK pathway and angiogenic-signaling pathways mediated by VEGFR and PDGFR are potent molecular targets in HCC therapy.

Sorafenib is an oral multi-kinase inhibitor that mainly targets Raf kinases and receptor tyrosine kinases associated with angiogenesis (VEGFR-2/-3, PDGFR- $\beta$ ). In a phase I study of sorafenib conducted in 69 patients with solid malignant tumors, diarrhea was the most commonly encountered treatment-related adverse event and the doselimiting toxicities were diarrhea, fatigue, and skin toxicities, namely, hand-foot syndromes and rash (Strumberg et al 2005). The maximum-tolerated dose was found to be $400 \mathrm{mg}$ bid continuous and the recommended dose for future studies was also determined to be $400 \mathrm{mg}$ bid continuous (Strumberg et al 2005). In regard to the efficacy, a partial response (PR) was observed in only one of 45 patients treated continuously with sorafenib at doses of $\geq 100 \mathrm{mg}$ bid, who was a patient HCC treated with the drug at $400 \mathrm{mg}$ bid continuous (Strumberg et al 2005). In this phase I study, 6 HCC patients were assessable for efficacy, of which one showed PR, 4 had stable disease (SD), and 1 showed progressive disease (PD) (Strumberg et al 2005). Based on these preclinical results and the results of the phase I study of sorafenib, a phase II study was performed in 137 patients with advanced HCC (Abou-Alfa et al 2006). Although the response rate was low, that is, $2.2 \%$, the time-to progression (TTP) and OS were promising; the median TTP was 4.2 months and the median OS was 9.2 months (Table 2). The grade 3/4 drugrelated toxicities included fatigue $(9.5 \%)$, diarrhea $(8.0 \%)$, and hand - foot skin reactions (5.1\%), all of which were manageable. In this phase II study, the relationship between the phosphorylated ERK levels in the tumor cells and the efficacy was examined, and no significant difference was found in the TTP between patients with higher staining intensity of phosphorylated ERK in the tumor cells and those with a lower staining intensity (Abou-Alfa et al 2006).

Based on these results, a large randomized controlled trial (RCT) of sorafenib versus placebo (the SHARP trial) was conducted in patients with advanced HCC (Llovet et al 2008). Six hundred two patients were randomized into 2 arms, the sorafenib arm and the placebo arm (Table 3). The TTP was 5.5 months for sorafenib and 2.8 months for placebo, and the hazard ratio in the sorafenib arm was 0.58 (95\% CI: 0.45-0.74; p < 0.001). The median OS was 10.7 months for sorafenib and 7.9 months for 


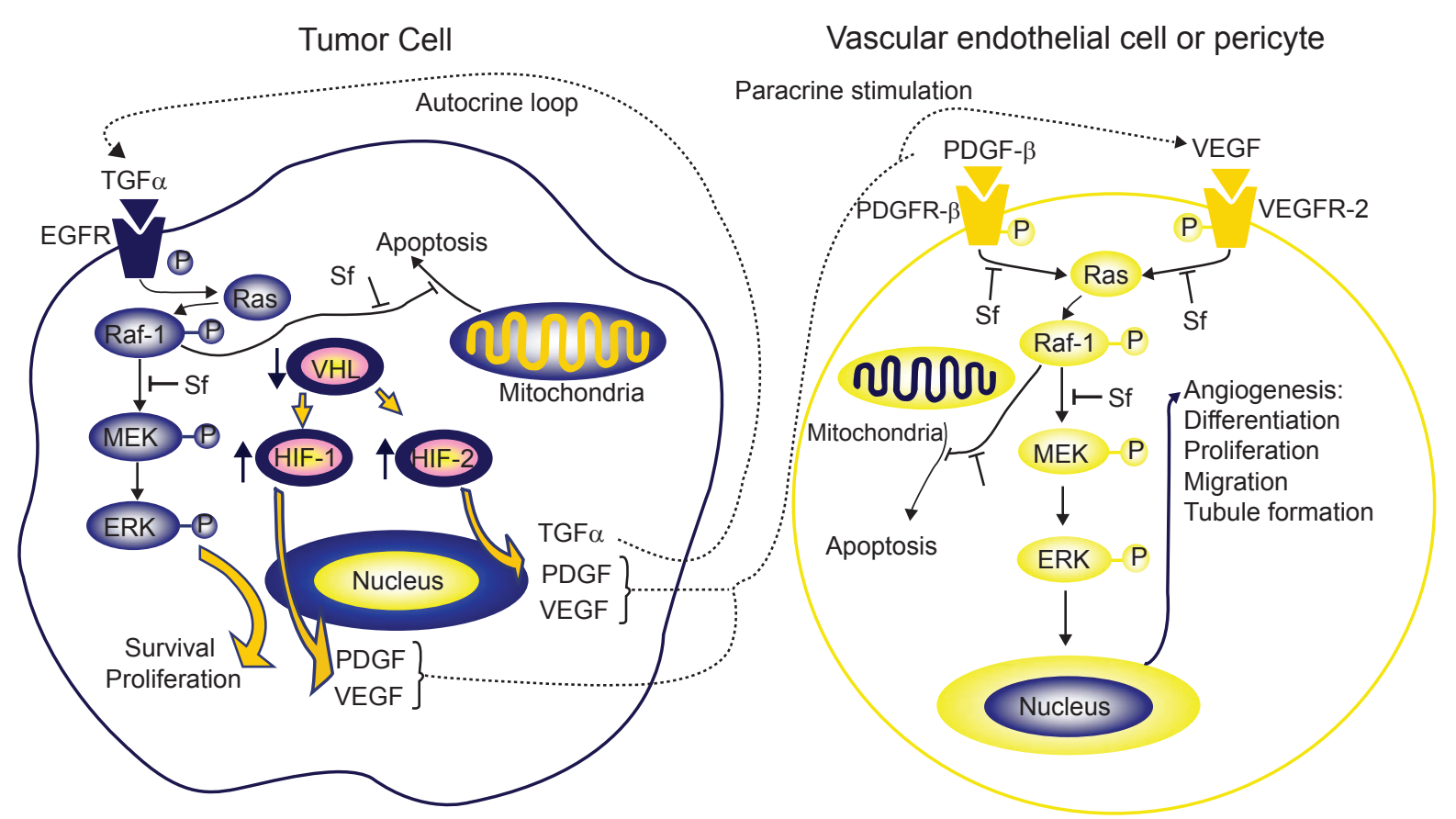

Figure 2 Mechanism of action of sorafenib. Sorafenib exerts a dual anticancer effect on the tumor and tumor vasculature by inhibiting Raf kinases including Raf-I as well as the receptor tyrosine kinases vascular endothelial growth factor receptor 2 (VEGFR2),VEGFR3, platelet-derived growth factor receptor (PDGFR), etc. Reproduced with permission from Gollob JA, Wilhelm S, Carter C, et al 2006. Role of Raf kinase in cancer: therapeutic potential of targeting the Raf/MEK/ERK signal transduction pathway. Semin Oncol, 33:392-406. Copyright @ 2006 Elsevier.

Abbreviation: Sf, sorafenib.

placebo, and the hazard ratio for OS in the sorafenib arm was 0.69 (95\% CI: $0.55-0.87 ; \mathrm{p}<0.001$ ) (Llovet et al 2008). This trial demonstrated a statistically significant improvement not only in the status of disease progression, but also in the survival of the patients in the sorafenib arm. The most frequently encountered drug-related serious adverse events were hand-hoot skin reaction, diarrhea and weight loss (Table 4). Thus, sorafenib is the first systemic chemotherapeutic agent to prolong survival in patients with advanced HCC.

\section{Clinical concerns related to the use of sorafenib in hepatocellular carcinoma}

In the SHARP trial, approximately $90 \%$ of the patients enrolled were from Europe or Australia (Table 3). The difference in the efficacy and safety was a concern in relation to the application of sorafenib as a global standard therapeutic agent for advanced $\mathrm{HCC}$, as the etiology and treatment strategies of HCC vary among regions in the world (Bruix et al 2001; Bruix and Sherman 2005; Ikai et al 2005; Kudo and Okanoue 2007). Therefore, to confirm the efficacy and safety of the drug in Asian populations, a RCT of sorafenib was conducted in the Asia-Pacific region (the Asia-Pacific trial) (Cheng et al 2008). The dose of sorafenib was the same as that in the SHARP trial, namely, $400 \mathrm{mg}$ bid continuous, and patients were randomized 2:1 to sorafenib or placebo (Table 3). The most frequently reported drug-related serious adverse events were hand-hoot skin reactions, diarrhea, hyperbilirubinemia and fatigue, similar to those observed in the SHARP trial (Table 4). The median OS of the primary endpoint was 6.5 months for sorafenib and 4.2 months for placebo, and the hazard ratio for OS in the sorafenib arm was 0.67 (95\% CI: 0.49-0.93; $p=0.0155)$ (Cheng et al 2008). The TTP was 2.8 months in the sorafenib arm and 1.4 months in the placebo arm, and the hazard ratio in the sorafenib arm was 0.58 (95\% CI: $0.42-0.80 ; p=0.0007)$. The etiology of $\mathrm{HCC}$ in the Asia-Pacific trial was different from that in the SHARP trial; HBV infection was observed in more than $70 \%$ of the patients in Asia (Table 3). However, the hazard ratios of OS and TTP were very similar to those in the SHARP trial, which indicated that efficacy is expected regardless of the difference in the etiology. Despite the equivalent hazard ratio for OS and TTP in the two RCTs, median OS and TTP were very poor in the Asia-Pacific trial compared with those in the SHARP trial, phase II and Japanese phase I studies. 
Table 2 Phase II study of sorafenib in the US and Europe and phase I study in Japan

\begin{tabular}{lll}
\hline Study & $\begin{array}{l}\text { Phase II study } \\
\text { (Abou-Alfa et al 2006) }\end{array}$ & $\begin{array}{l}\text { Japanese Phase I study } \\
\text { (Furuse et al 2008) }\end{array}$ \\
\hline $\mathrm{n}$ & 137 & 27 \\
Child-Pugh A/B & $98 / 38$ & $13 / 14$ \\
Dose & $400 \mathrm{mg} \mathrm{bid}$ & $200,400 \mathrm{mg}$ bid \\
Partial response & $3(2.2 \%)$ & $1(3.7 \%)$ \\
Stable disease & $54(39.4 \%)$ & $21(77.8 \%)$ \\
Disease control rate & $41.6 \%$ & $81.5 \%$ \\
Progressive disease & $48(35.0 \%)$ & $3(\mathrm{II} .1 \%)$ \\
Not assessed & $32(23.4 \%)$ & $2(7.4 \%)$ \\
Median time to progression & $4.2 \mathrm{mo}$ & $4.9 \mathrm{mo}$ \\
Median overall survival & $9.2 \mathrm{mo}$ & $15.6 \mathrm{mo}$ \\
\hline
\end{tabular}

This disadvantage was due to the difference in the patient characteristics, such as the poorer performance status $(69 \%$ of ECOG PS [Eastern Cooperative Oncology Group Performance Status]) and more advanced stage of the cancer ( $96 \%$ of BCLC [Barcelona Clinic Liver Cancer staging classification] stage $\mathrm{C}, 52 \%$ of lung metastases). In conclusion, from the SHARP trial and the Asia-Pacific trial, while the difference in the etiology of HCC is not of critical concern, the advanced stage of the disease at diagnosis and a poor PS may be among the main reasons for the poor outcomes of sorafenib treatment.

There is another important issue of whether sorafenib can be used in patients with Child-Pugh class B disease, or moderate liver dysfunction, because patients enrolled in the SHARP trial were limited to those with good liver function classified into Child-Pugh A. In Japan, a phase I study of sorafenib was conducted to investigate the pharmacokinetics, safety and efficacy of the drug in Japanese patients with advanced HCC; there was an equal number of patients with Child-Pugh A and B disease (Furuse et al 2008). The results revealed a safety profile similar to that in the RCTs of sorafenib and the same recommended dose of $400 \mathrm{mg}$ bid. The response rate was $3.7 \%$, and the median TTP and OS were 4.9 months and 15.6 months, respectively (Table 2) (Furuse et al 2008). In regard to the difference in the pharmacokinetics between patients with Child-Pugh A and B, although both areas under the concentration - time curve for 0 to $12 \mathrm{~h}$ and the maximal concentration at steady state were slightly lower in the Child - Pugh class B patients than in the Child-Pugh class A patients. There was no major difference in the incidence and grade of drug-related adverse

Table 3 Patient background in randomized controlled trials of sorafenib versus placebo in the SHARP trial and Asia-Pacific trial

\begin{tabular}{|c|c|c|c|c|}
\hline & \multicolumn{2}{|c|}{ SHARP study (Llovet et al 2008) } & \multicolumn{2}{|c|}{ Asia-Pacific study (Cheng et al 2008) } \\
\hline & Sorafenib & Placebo & Sorafenib & Placebo \\
\hline $\mathrm{N}$ & 299 & 303 & 150 & 76 \\
\hline Median age & 64.9 & 66.3 & 51 & 52 \\
\hline Male & $87 \%$ & $87 \%$ & $85 \%$ & $87 \%$ \\
\hline Region: Europe and Australia & $88 \%$ & $87 \%$ & - & - \\
\hline $\mathrm{HCV} / \mathrm{HBV} / \mathrm{alcohol}$ & $29 / 19 / 26 \%$ & $27 / 18 / 26 \%$ & $71 / 11 / N A \%$ & 78/4/NA\% \\
\hline ECOG PS $0 / 1 / 2$ & $54 / 38 / 8 \%$ & $54 / 39 / 7 \%$ & $25 / 69 / 5 \%$ & $28 / 67 / 5 \%$ \\
\hline $\mathrm{BCLC}$ stage $\mathrm{B} / \mathrm{C}$ & $18 / 82 \%$ & $17 / 83 \%$ & $4 / 96 \%$ & $4 / 96 \%$ \\
\hline Macroscopic vascular invasion & $36 \%$ & $41 \%$ & $36 \%$ & $34 \%$ \\
\hline Extrahepatic spread & $53 \%$ & $50 \%$ & $69 \%$ & $68 \%$ \\
\hline Lymph nodes & $30 \%$ & $21 \%$ & $31 \%$ & $34 \%$ \\
\hline Lung & $22 \%$ & $19 \%$ & $52 \%$ & $45 \%$ \\
\hline Child-Pugh A & $95 \%$ & $98 \%$ & $97 \%$ & $97 \%$ \\
\hline
\end{tabular}

Abbreviations: $\mathrm{HCV}$, hepatitis C virus; $\mathrm{HBV}$, hepatitis B virus; PS, performance status; $\mathrm{BCLC}$ stage, barcelona clinic liver cancer staging classification; $\mathrm{ECOG}$ PS, eastern cooperative oncology group performance status. 
Table 4 Severe drug-related serious adverse events in the SHARP trial and Asia-Pacific trial

\begin{tabular}{|c|c|c|c|c|c|}
\hline \multirow[b]{2}{*}{ Grade $3 / 4$ toxicity } & \multicolumn{3}{|c|}{ SHARP study (Llovet et al 2008) } & \multicolumn{2}{|c|}{ Asia-Pacific study (Cheng et al 2008) } \\
\hline & Sorafenib & Placebo & $\mathrm{p}$-value & Sorafenib & Placebo \\
\hline Hand-foot skin reaction & $8 \%$ & $<1 \%$ & $<0.001$ & $10 \%$ & $0 \%$ \\
\hline Diarrhea & $8 \%$ & $2 \%$ & $<0.001$ & $6 \%$ & $0 \%$ \\
\hline Hyperbilirubinemia & - & - & - & $3 \%$ & $3 \%$ \\
\hline Fatigue & $4 \%$ & $<4 \%$ & I & $3 \%$ & $1 \%$ \\
\hline Weight loss & $2 \%$ & $0 \%$ & 0.03 & - & - \\
\hline Hypertension & $2 \%$ & $1 \%$ & 0.28 & - & - \\
\hline Total & $13 \%$ & $9 \%$ & - & $9 \%$ & $1 \%$ \\
\hline
\end{tabular}

events between the Child-Pugh class A and B groups, but hypertension, hand - foot skin reactions, and rash were more frequently observed in the Child-Pugh class B group than in the Child-Pugh class A group. In a phase I study of sorafenib for solid tumors and hematologic malignancies in patients with hepatic or renal dysfunction, it was observed that 9 out of 12 events in patients with liver dysfunction as the dose-limiting toxicity were increase in the serum bilirubin level (Miller et al 2007).

Comparison of the efficacy and safety between Child-Pugh class A and B patients was also examined in a phase II study in HCC patients (Abou-Alfa et al 2008a). The pharmacokinetic and overall toxic profiles were similar in both groups. However, adverse events related to liver cirrhosis, such as bilirubin increase, ascites and encephalopathy, occurred more frequently in Child-Pugh class B than class A patients. Furthermore, the TTP and OS appeared to be worse in the Child-Pugh class B patients than in the Child-Pugh class A patients. The Child-Pugh class B group is generally heterogeneous with scores ranging from 7 to 9 (Pugh et al 1973). The indications of sorafenib in Child-Pugh B patients should be carefully considered and more data are required to confirm the safety and efficacy of sorafenib in practice and in clinical trials in these patients in the future.

\section{Conclusions and future directions of chemotherapy for HCC}

In patients with advanced HCC, the survival benefit of sorafenib has been demonstrated in a RCT for the first time, and the drug has already been approved for the treatment of HCC. However, the efficacy remains moderate, and

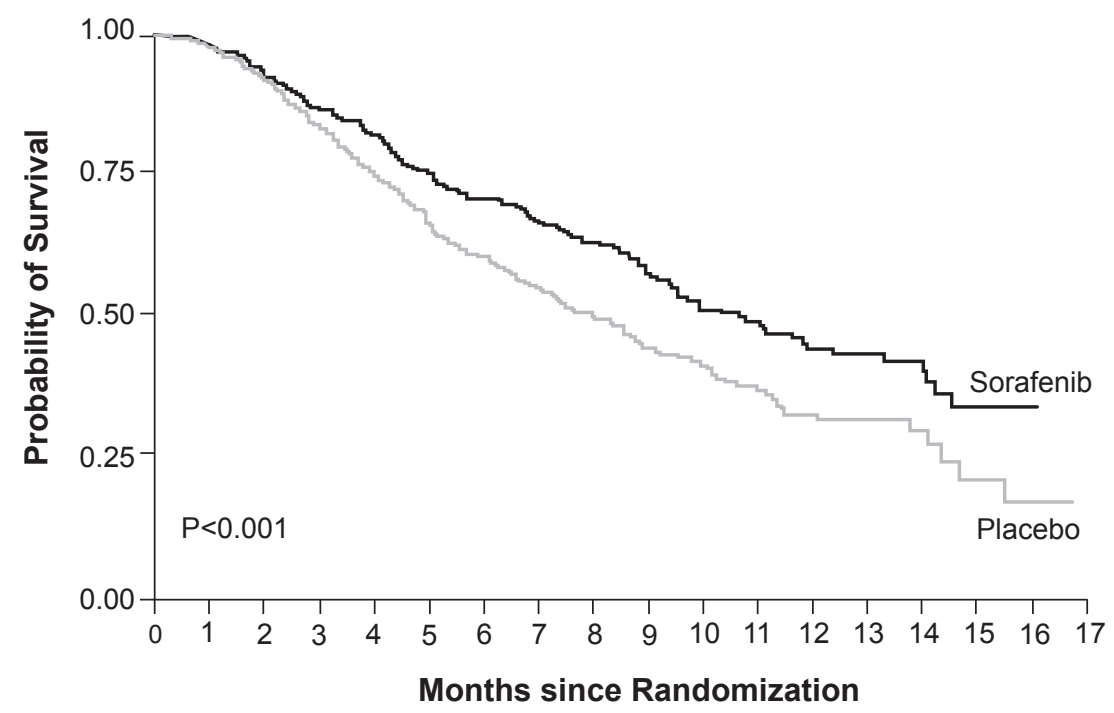

No. at Risk

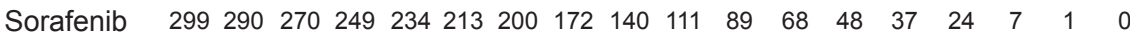

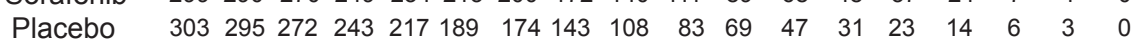

Figure 3 Survival benefit was confirmed in the SHARP trial; the median overall survival was 10.7 months in the sorafenib group as compared with 7.9 months in the placebo group (hazard ratio for death in the sorafenib group, $0.69 ; 95 \% \mathrm{Cl}: 0.55-0.87$ ). Reproduced with permission from Llovet JM, Ricci S, MazzaferroV, et al 2008. Sorafenib in advanced hepatocellular carcinoma. N Engl J Med, 359:378-90. Copyright (C) Massachusetts Medical Society. All rights reserved. 


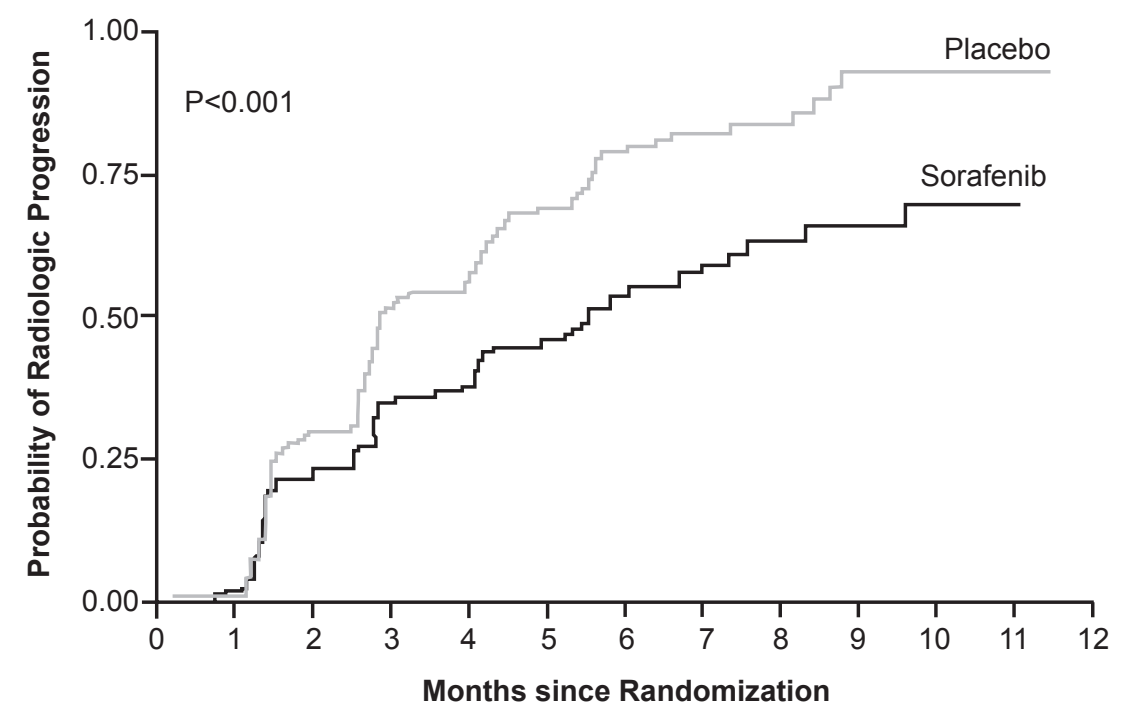

No. at Risk

$\begin{array}{llllllllllllll}\text { Sorafenib } & 299 & 267 & 155 & 101 & 91 & 65 & 37 & 23 & 18 & 10 & 4 & 2 & 0\end{array}$

$\begin{array}{llllllllllllll}\text { Placebo } & 303 & 275 & 142 & 78 & 62 & 41 & 21 & 11 & 10 & 3 & 1 & 1 & 0\end{array}$

Figure 4 Median time to progression (TTP) was 5.5 months in the sorafenib group as compared with 2.8 months in the placebo group (hazard ratio for progression in the sorafenib group, $0.58 ; 95 \% \mathrm{Cl}: 0.45-0.74)$ in the SHARP trial. There was a statistically significant difference in the TTP between the two groups. Reproduced with permission from Llovet JM, Ricci S, Mazzaferro V, et al 2008. Sorafenib in advanced hepatocellular carcinoma. N Engl J Med, 359:378-90. Copyright (C) Massachusetts Medical Society. All rights reserved.

other promising agents need to be developed for further prolonging the survival in HCC patients. Sunitinib, an oral, multitargeted tyrosine kinase inhibitor, has shown promising activity against HCC in phase II studies, with a median progression-free survival (PFS) of about 4 months and median OS of about 10 months (Faivre et al 2007; Zhu et al 2008). Bevacizumab, a recombinant, humanized monoclonal antibody that targets VEGF, has been investigated in phase II studies of bevacizumab alone or the agent administered in combination with erlotinib (Thomas et al 2007; Siegel et al 2008). The response rates in these studies were moderate, ranging from $10 \%$ to $20 \%$. The median PFS achieved was very promising, ranging from 5.3 to 9.0 months, especially for the combination of bevacizumab and erlotinib. On the other hand, enhancement of the efficacy of sorafenib by its use in combination regimens has also been investigated, eg, a randomized phase II study of sorafenib plus doxorubicin showed encouraging outcomes in terms of the TTP and OS, with a median TTP of 8.5 months and median OS of 14.0 months (Abou-Alfa et al 2008b). Some agents selectively targeting VEGFR or mTOR are currently being investigated for various solid tumors, and are eventually expected to also be applied for the treatment of HCC.

There would be various ways in development of new systemic therapeutic regimen after the establishment of standard therapy using sorafenib. One of them is the use of sorafenib-based combined therapy as first-line therapy. Combination with cytotoxic agents would be investigated, but the hepatic toxicity may be a concern, as previous many cytotoxic chemotherapeutic regimens failed to prolong the survival in HCC patients. Since various cellular signaling pathways are implicated in hepatocarcinogenesis, combination with other molecular-targeted agents with different mechanisms of action would be promising, but the toxicity may again be a concern. Another issue of interest is secondline treatment in patients with sorafenib-refractory disease. In HCC patients with good liver function, prolonged survival would be expected even in cases showing disease progression after sorafenib treatment; eg, a survival time of 5.2 months was obtained after disease progression in the SHARP trial. In a Japanese phase I study of sorafenib, despite the median TTP being only 4.9 months, the median OS was relatively good, at 15.6 months. Various treatments might influence the survival after sorafenib failure. Therefore, it would be possible to develop post-sorafenib treatments, and second-line treatment is important to prolong the survival.

Although sorafenib is the first agent shown to improve the survival of patients with advanced HCC and is generally well tolerated, diverse results in terms of the safety and the efficacy have been obtained; some patients show severe toxicities, such as hand - foot skin reactions, and some patients show very short survival. Population differences in gene expressions 
could contribute to the differences in the susceptibility of HCC to drug treatments. Genome-wide association studies for local or distant genetic variants correlated with the differentially expressed genes led to identification of significant associations with one or more single-nucleotide polymorphisms (SNPs) (Stranger et al 2007; Zhang et al 2008). In order to identify patients who will benefit from sorafenib, attempts must be made, by pharmacogenomic studies, to identify biomarkers and/or SNPs that would predict the efficacy, toxicity and resistance to sorafenib.

Sorafenib is currently established as a drug for prolonging the survival in HCC patients with metastatic disease or TACE-refractory disease who are not suitable candidates for local treatments. The FDA approved sorafenib for use in the treatment of patients with unresectable HCC. Therefore, it can also be used in combination with local therapies, such as local ablation therapy or TACE. Although interest has also been focused on the use of the drug as adjuvant treatment after local treatments like surgical resection, ablation therapy and TACE, no adjuvant treatments after local therapies have been established to date in HCC. In Japan and Korea, a large RCT of sorafenib as post-TACE adjuvant therapy is ongoing. Rather than gross advanced tumors, tiny residual tumors after these local treatments seem to be more effectively treated by cytostatic agents like sorafenib. Furthermore, there is the possibility of preventing progression from precancerous lesions like dysplastic nodules associated with cirrhotic liver to cancer using growth factor inhibitors. In the future, investigation of sorafenib or other molecular-targeted therapies is also expected in the adjuvant or prophylactic setting.

\section{Disclosures}

The author has no conflicts of interest to disclose.

\section{References}

Abou-Alfa G, Johnson P, Knox J, et al. 2008b. Preliminary results from a Phase II, randomized, double-blind study of sorafenib plus doxorubicin versus placebo plus doxorubicin in patients with advanced hepatocellular carcinoma. Eur J Cancer, 5(Suppl):259, \#3500.

Abou-Alfa GK, Amadori D, Santoro A, et al. 2008a. Is sorafenib (S) safe and effective in patients (pts) with hepatocellular carcinoma (HCC) and Child-Pugh B (CPB) cirrhosis? Proc Am Soc Clin Oncol, 26: 217s, \#4518.

Abou-Alfa GK, Schwartz L, Ricci S, et al. 2006. Phase II study of sorafenib in patients with advanced hepatocellular carcinoma. J Clin Oncol, 24:4293-300.

Avila MA, Berasain C, Sangro B, et al. 2006. New therapies for hepatocellular carcinoma. Oncogene, 25:3866-84.

Boucher E, Corbinais S, Brissot P, et al. 2002. Treatment of hepatocellular carcinoma (HCC) with systemic chemotherapy combining epirubicin, cisplatinum and infusional 5-fluorouracil (ECF regimen). Cancer Chemother Pharmacol, 50:305-8.
Bruix J, Sherman M, Llovet JM, et al. 2001. Clinical management of hepatocellular carcinoma. Conclusions of the Barcelona-2000 EASL conference. European Association for the Study of the Liver. J Hepatol, $35: 421-30$.

Bruix J, Sherman M. 2005. Management of hepatocellular carcinoma. Hepatology, 42:1208-36.

Chang YS, Adnane J, Trail PA, et al. 2007. Sorafenib (BAY 43-9006) inhibits tumor growth and vascularization and induces tumor apoptosis and hypoxia in RCC xenograft models. Cancer Chemother Pharmacol, 59:561-74.

Chao Y, Li CP, Chau GY, et al. 2003. Prognostic significance of vascular endothelial growth factor, basic fibroblast growth factor, and angiogenin in patients with resectable hepatocellular carcinoma after surgery. Ann Surg Oncol, 10:355-62.

Chen J, Siddiqui A. 2007. Hepatitis B virus X protein stimulates the mitochondrial translocation of Raf-1 via oxidative stress. J Virol, 81:6757-60.

Cheng AL, Kang Y, Chen Z, et al. 2008. Randomized phase III trial of sorafenib versus placebo in Asian patients with advanced hepatocellular carcinoma. Proc Am Soc Clin Oncol, 26:215s, \#4509.

Colleoni M, Nole F, Di Bartolomeo M, et al. 1992. Mitoxantrone in patients affected by hepatocellular carcinoma with unfavorable prognostic factors. Oncology, 49:139-42.

Davies H, Bignell GR, Cox C, et al. 2002. Mutations of the BRAF gene in human cancer. Nature, 417:949-54.

El-Serag HB, Mason AC. 1999. Rising incidence of hepatocellular carcinoma in the United States. N Engl J Med, 340:745-50.

Faivre SJ, Raymond E, Douillard J, et al. 2007. Assessment of safety and drug-induced tumor necrosis with sunitinib in patients (pts) with unresectable hepatocellular carcinoma (HCC). Proc Am Soc Clin Oncol, 25:149s, \#3546.

Furuse J, Ishii H, Nakachi K, et al. 2008. Phase I study of sorafenib in Japanese patients with hepatocellular carcinoma. Cancer Sci, 99:159-65.

Giambartolomei S, Covone F, Levrero M, et al. 2001. Sustained activation of the Raf/MEK/Erk pathway in response to EGF in stable cell lines expressing the Hepatitis C Virus (HCV) core protein. Oncogene, 20:2606-10.

Gollob JA, Wilhelm S, Carter C, et al. 2006. Role of Raf kinase in cancer: therapeutic potential of targeting the Raf/MEK/ERK signal transduction pathway. Semin Oncol, 33:392-406.

Hackel PO, Zwick E, Prenzel N, et al. 1999. Epidermal growth factor receptors: critical mediators of multiple receptor pathways. Curr Opin Cell Biol, 11:184-9.

Hood JD, Bednarski M, Frausto R, et al. 2002. Tumor regression by targeted gene delivery to the neovasculature. Science, 296:2404-7.

Huynh H, Nguyen TT, Chow KH, et al. 2003. Over-expression of the mitogen-activated protein kinase (MAPK) kinase (MEK)-MAPK in hepatocellular carcinoma: its role in tumor progression and apoptosis. BMC Gastroenterol, 3:19-39.

Hwang YH, Choi JY, Kim S, et al. 2004. Over-expression of c-raf-1 proto-oncogene in liver cirrhosis and hepatocellular carcinoma. Hepatol Res, 29:113-21.

Ikai I, Arii S, Ichida T, Okita K, et al. 2005. Report of the 16th follow-up survey of primary liver cancer. Hepatol Res, 32:163-72.

Ikeda M, Okusaka T, Ueno H, et al. 2005. A phase II trial of continuous infusion of 5-fluorouracil, mitoxantrone, and cisplatin for metastatic hepatocellular carcinoma. Cancer, 103:756-62.

Ito Y, Sasaki Y, Horimoto M, et al. 1998. Activation of mitogen-activated protein kinases/extracellular signal-regulated kinases in human hepatocellular carcinoma. Hepatology, 27:951-8.

Kimura ET, Nikiforova MN, Zhu Z, et al. 2003. High prevalence of BRAF mutations in thyroid cancer: genetic evidence for constitutive activation of the RET/PTC-RAS-BRAF signaling pathway in papillary thyroid carcinoma. Cancer Res, 63:1454-7.

Kudo M, Okanoue T; Japan Society of Hepatology. 2007. Management of hepatocellular carcinoma in Japan: consensus-based clinical practice manual proposed by the Japan Society of Hepatology. Oncology, 72(Suppl 1):2-15. 
Lai CL, Wu PC, Chan GC, et al. 1988. Doxorubicin versus no antitumor therapy in inoperable hepatocellular carcinoma. A prospective randomized trial. Cancer, 62:479-83.

Leung TW, Patt YZ, Lau WY, et al. 1999. Complete pathological remission is possible with systemic combination chemotherapy for inoperable hepatocellular carcinoma. Clin Cancer Res, 5:1676-81.

Liu L, Cao Y, Chen C, Zhang X, et al. 2006. Sorafenib blocks the RAF/MEK/ ERK pathway, inhibits tumor angiogenesis, and induces tumor cell apoptosis in hepatocellular carcinoma model PLC/PRF/5. Cancer Res, 66:11851-8.

Llovet JM, Ricci S, Mazzaferro V, et al. 2008. Sorafenib in advanced hepatocellular carcinoma. N Engl J Med, 359:378-90.

McGlynn KA, Tsao L, Hsing AW, et al. 2001. International trends and patterns of primary liver cancer. Int J Cancer, 94:290-6.

Miller AA, Murry DJ, Owzar K, et al. 2007. Pharmacokinetic (PK) and phase I study of sorafenib $(\mathrm{S})$ for solid tumors and hematologic malignancies in patients with hepatic or renal dysfunction (HD or RD): CALGB 60301. Proc Am Soc Clin Oncol, 25:147s, \#3538.

Miura H, Miyazaki T, Kuroda M, et al. 1997. Increased expression of vascular endothelial growth factor in human hepatocellular carcinoma. J Hepatol, 27:854-61.

Oka H, Chatani Y, Hoshino R, et al. 1995. Constitutive activation of mitogen-activated protein (MAP) kinases in human renal cell carcinoma. Cancer Res, 55:4182-7.

Parkin DM, Bray F, Ferlay J, et al. 2005. Global cancer statistics, 2002. CA Cancer J Clin, 55:74-108.

Patt YZ, Hassan MM, Lozano RD, et al. 2003. Phase II trial of systemic continuous fluorouracil and subcutaneous recombinant interferon Alfa-2b for treatment of hepatocellular carcinoma. J Clin Oncol, 21:421-7.

Poon RT, Ho JW, Tong CS, et al. 2004. Prognostic significance of serum vascular endothelial growth factor and endostatin in patients with hepatocellular carcinoma. Br J Surg, 91:1354-60.

Pugh RNH, Murray-Lyon IM, Dawson JL, et al. 1973. Transection of the esophagus for bleeding esophageal varices. Br J Surg, 60:646-54.

Schmidt CM, McKillop IH, Cahill PA, et al. 1997. Increased MAPK expression and activity in primary human hepatocellular carcinoma. Biochem Biophys Res Commun, 236:54-8.
Siegel AB, Cohen EI, Ocean A, et al. 2008. Phase II trial evaluating the clinical and biologic effects of bevacizumab in unresectable hepatocellular carcinoma. J Clin Oncol, 26:2992-8.

Stranger BE, Nica AC, Forrest MS, et al. 2007. Population genomics of human gene expression. Nat Genet, 39:1217-24.

Strumberg D, Richly H, Hilger RA, et al. 2005. Phase I clinical and pharmacokinetic study of the Novel Raf kinase and vascular endothelial growth factor receptor inhibitor BAY 43-9006 in patients with advanced refractory solid tumors. J Clin Oncol, 23:965-72.

Thomas MB, Chadha R, Iwasaki M, et al. 2007. The combination of bevacizumab (B) and erlotinib (E) shows significant biological activity in patients with advanced hepatocellular carcinoma (HCC). Proc Am Soc Clin Oncol, 25:214s, \#4567.

Wilhelm S, Carter C, Lynch M, et al. 2006. Discovery and development of sorafenib: a multikinase inhibitor for treating cancer. Nat Rev Drug Discov, 5:835-44.

Wilhelm SM, Carter C, Tang L, et al. 2004. BAY 43-9006 exhibits broad spectrum oral antitumor activity and targets the RAF/MEK/ERK pathway and receptor tyrosine kinases involved in tumor progression and angiogenesis. Cancer Res, 64:7099-109.

Woodburn JR. 1999. The epidermal growth factor receptor and its inhibition in cancer therapy. Pharmacol Ther, 82:241.

Yamaguchi R, Yano H, Iemura A, et al. 1998. Expression of vascular endothelial growth factor in human hepatocellular carcinoma. Hepatology, 28:68-77.

Yeo W, Mok TS, Zee B, et al. 2005. A randomized phase III study of doxorubicin versus cisplatin/interferon alpha-2b/doxorubicin/ fluorouracil (PIAF) combination chemotherapy for unresectable hepatocellular carcinoma. J Natl Cancer Inst, 97:1532-8.

Zhang W, Duan S, Kistner EO, et al. 2008. Evaluation of genetic variation contributing to differences in gene expression between populations. Am J Hum Genet, 82:631-40.

Zhu AX, Sahani DV, di Tomaso E, et al. 2008. Sunitinib monotherapy in patients with advanced hepatocellular carcinoma (HCC): Insights from a multidisciplinary phase II study. Proc Am Soc Clin Oncol, 26:218s, \#4521. 